\title{
Evaluation of Collaterals and Clot Burden Using Time-Resolved C-Arm Conebeam CT Angiography in the Angiography Suite: A Feasibility Study
}

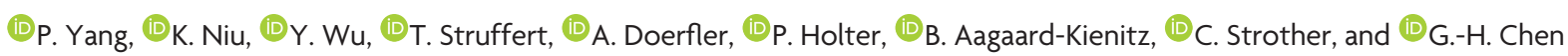

\begin{abstract}
BACKGROUND AND PURPOSE: The assessment of collaterals and clot burden in patients with acute ischemic stroke provides important information about treatment options and clinical outcome. Time-resolved C-arm conebeam CT angiography has the potential to provide accurate and reliable evaluations of collaterals and clot burden in the angiographic suite. Experience with this technique is extremely limited, and feasibility studies are needed to validate this technique. Our purpose was to present such a feasibility study.
\end{abstract}

MATERIALS AND METHODS: Ten C-arm conebeam CT perfusion datasets from 10 subjects with acute ischemic stroke acquired before endovascular treatment were retrospectively processed to generate time-resolved conebeam CTA. From time-resolved conebeam CTA, 2 experienced readers evaluated the clot burden and collateral flow in consensus by using previously reported scoring systems and assessed the clinical value of this novel imaging technique independently. Interobserver agreement was analyzed by using the intraclass correlation analysis method.

RESULTS: Clot burden and collateral flow can be assessed by using the commonly accepted scoring systems for all eligible cases. Additional clinical information (eg, the quantitative dynamic information of collateral flow) can be obtained from this new imaging technique. Two readers agreed that time-revolved C-arm conebeam CTA is the preferred method for evaluating the clot burden and collateral flow compared with other conventional imaging methods.

CONCLUSIONS: Comprehensive evaluations of clot burden and collateral flow are feasible by using time-resolved C-arm conebeam CTA data acquired in the angiography suite. This technique further enriches the imaging tools in the angiography suite to enable a "one-stopshop" imaging workflow for patients with acute ischemic stroke.

ABBREVIATIONS: AIS = acute ischemic stroke; $\mathrm{CBCT}=$ conebeam $\mathrm{CT} ; \mathrm{CBCTA}=$ conebeam CTA; $\mathrm{CBCTP}=$ conebeam CTP; tMIP = temporal maximum-intensityprojection; LVO = large-vessel occlusion

$\mathbf{R}_{b}^{\mathrm{c}}$ ecent studies have shown that the status of collaterals and clot burden evaluated with multidetector row CT can be used as 2 independent parameters to predict both the success of revascularization and the ultimate clinical outcome of patients with acute ischemic stroke (AIS). ${ }^{1-4}$ Thus, these 2 parameters can be used as potentially valuable metrics for the selection of appropriate candidates for endovascular thrombectomy. ${ }^{5,6}$ However, the acqui-

Received July 20, 2016; accepted after revision November 7.

From the Department of Neurosurgery (P.Y.), Changhai Hospital, Second Military Medical University, Shanghai, China; Departments of Radiology (P.Y., B.A.-K., C.S., G.-H.C.) and Medical Physics (K.N., Y.W., G.-H.C.), School of Medicine and Public Health, University of Wisconsin-Madison, Madison, Wisconsin; and Department of Neuroradiology (T.S., A.D., P.H.), University of Erlangen-Nuremberg, Erlangen, Germany.

This work was partially funded from a quantum grant (U01 EB201183) from the National Institute of Biomedical Imaging and Bioengineering. We also acknowledge the funding of the Medical Valley National Leading Edge Cluster, Erlangen, Germany, Diagnostic Imaging Network, subproject BD 16, research grant No. 13EX1212G. Dr Chen received funding support from Siemens Healthineers. Dr Strother received funding support from both Siemens Healthineers and the Department of Radiology, University of Wisconsin-Madison. sition of multidetector row CT images to assess clot burden and collaterals may delay the time from stroke onset to revascularization; this delay is primarily due to the time needed to transfer patients among different sites in a clinical facility. As a result, acquisition of complete anatomic and physiologic imaging for a comprehensive diagnosis and evaluation of eligible patients becomes a reluctant option in the current clinical practice. It would be ideal to acquire all the needed imaging information for AIS directly in an angiography suite (ie, a "one-stop-shop" imaging workflow in the angiography suite), to avoid delay while performing comprehensive evaluations of patients with AIS.

Recently, with a C-arm conebeam CT (CBCT) acquisition

Please address correspondence to Charles Strother, MD, Department of Radiology, University of Wisconsin, 600 Highland Ave, Box 3252 Clinical Science Center, Madison, WI 53792; e-mail: Strother@wisc.edu; Guang-Hong Chen, PhD, Department of Radiology and Department of Medical Physics, UW-Madison, Madison, Wisconsin; e-mail: gchen7@wisc.edu

-* Indicates open access to non-subscribers at www.ajnr.org

http://dx.doi.org/10.3174/ajnr.A5072 
platform in the angiographic suite, the feasibility of acquiring dynamic CBCT perfusion (CBCTP) maps in the angiography suite has been demonstrated both in animals and in human subjects. $^{7,8}$ From the acquired CBCT perfusion dataset, time-resolved conebeam CT angiography (CBCTA) can also be generated to provide accurate diagnosis of large-vessel occlusions (LVOs). ${ }^{9,10}$ In this study, we hypothesized that the use of timeresolved CBCTA would facilitate the evaluation of collateral status and clot burden in patients with AIS. This hypothesis was tested with the time-resolved CBCTA generated by novel imageprocessing algorithms from a CBCTP acquisition in the angiography suite. ${ }^{1,12}$ The ability to accurately and reliably assess collateral status and clot burden by using time-resolved CBCTA would, in our opinion, further enrich the environment of the angiography suite as a one-stop-shop for AIS care.

\section{MATERIALS AND METHODS \\ Patient Selection}

Under an approved ethics committee protocol at University of Erlangen-Nuremberg, 17 consecutive patients with AIS with suspected LVO underwent both conventional pretreatment imaging (multidetector row CTP or MRP) and dynamic C-arm CBCTP examinations. During treatment procedures, these patients also underwent conventional DSA imaging as part of the standard of care. All patient imaging and data collection was performed at the University Hospital of the University of Erlangen-Nuremberg. Ten of these subjects had C-arm CBCTP datasets acquired before endovascular treatment. Data from these subjects were completely anonymized and shared with the team at the University of Wisconsin School of Medicine and Public Health. These are the subjects included in this study. These subjects had 4 ICA occlusions, 3 M1 occlusions, 2 M2 occlusions, and 1 basilar trunk occlusion. Details of data acquisition and data postprocessing follow below.

\section{Data Acquisition}

The details of the technique for obtaining the CBCTP data have been reported elsewhere. ${ }^{9,10}$ Briefly, the dynamic C-arm CBCTP data were acquired by using a biplane flat detector angiographic system (Axiom Artis zee; Siemens, Erlangen, Germany). Contrast was injected into a peripheral vein with a dual-syringe angiographic power injector (Accutron HP-D; Medtron, Saarbrücken, Germany). Sixty milliliters of contrast material (iopamidol, Imeron 350; Bracco, Milan, Italy) was injected at a rate of $5 \mathrm{~mL} / \mathrm{s}$ followed by a $60-\mathrm{mL}$ saline flush. Nine bidirectional rotational scans ( 5 forward rotations and 4 reverse rotations) were obtained for each subject; contrast was injected 5 seconds after the start of the acquisition so that the first 2 rotations served as the nonenhanced (mask) images, while the following 7 were contrast-enhanced (fill) images.

\section{Image Postprocessing}

$3 \mathrm{D}$ isotropic filtered back-projection image volume for each rotation was reconstructed and coregistered with the proprietary software of the vendor. To reduce noise and improve temporal resolution and temporal sampling density, we used Prior Image Constrained Compressed Sensing and Temporal Resolution and
Sampling Recovery techniques ${ }^{1,12}$ to generate time-resolved image volumes with better image quality and a half-second temporal resolution. Then, temporal maximum-intensity-projection (tMIP) image volumes were generated from time-resolved image volumes by assigning each image voxel the maximum value along the temporal direction. Both time-resolved and tMIP image volumes were imported into a Leonardo workstation (Siemens) for future measurement and evaluation.

\section{Image Evaluation}

Clot burden and collateral flow were evaluated by 2 experienced raters ( 30 and 8 years of experience in neurointervention) by consensus. Temporal MIP images were used to detect the proximal and distal sites of vessel occlusion to score the clot burden, ${ }^{13}$ measure the thrombus length and size of distal vessel, and evaluate collateral status. Collateral status was evaluated by using 3 commonly used scoring systems. ${ }^{4,13,14}$ Time-resolved image volumes were also displayed in MIP mode and used to evaluate the flow direction (antegrade or retrograde) of the MCA occlusions and also to generate time-density curves for selected symmetric measuring points on the bilateral MCA branches. From these timedensity curves, the differences of time-to-peak and peak density between the lesion and normal sides could be quantified. These parameters were of interest because they may reflect the differences of flow velocity and volume, respectively. The criterion standard used to identify the site of occlusion was the clinician's report of the 2D DSAs, which were performed at the start of treatment.

\section{Statistics}

Intraclass correlation analysis was performed by using SPSS, Version 20.0 (IBM, Armonk, New York). The Cronbach $\alpha$ coefficients were calculated to assess the interrater agreement on the subjective evaluation of the capability and potential of this novel technique. Alpha values were interpreted according to the following criteria: unacceptable $(\alpha<.5)$, poor $(0.5 \leq \alpha<.6)$, fair $(0.6 \leq$ $\alpha<.7)$, good $(0.7 \leq \alpha<.9)$, and excellent $(\alpha \geq .9)$.

\section{RESULTS}

The full extent of vessel occlusion (ie, the proximal and distal occlusion sites) could be determined by using tMIP images for all 10 patients. In 7 of these patients, the length of the thrombus could be measured ( 7 with terminal ICA, M1, basilar trunk, or M2 occlusions) and the measured lengths ranged from 5.9 to 19.9 $\mathrm{mm}$. In the 3 patients with cervical or cavernous ICA occlusion, thrombus length could not be measured due to the tortuous course of the ICA and the presence of streaking artifacts over the cavernous or cervical portions of the artery. Clot burden could thus be qualitatively evaluated for 6 patients by using a clot-burden score designed specifically for LVOs in the anterior circulation. ${ }^{13}$ The diameter of the occluded vessel distal to the thrombus could be measured for all patients (Table 1).

The evaluation of collateral flow was successfully performed for 7 patients with LVOs in the anterior circulation (4 ICAs, 3 M1s). In all of these patients, the extent of collateral flow could be assessed by using the tMIP images, regardless of which scoring system was used (Table 2). ${ }^{4,13,14}$ Antegrade flow into the down- 
Table 1: Evaluation of clot burden and distribution using time-resolved C-arm CBCTA data

\begin{tabular}{|c|c|c|c|c|c|c|}
\hline Case & Side & $\begin{array}{c}\text { Proximal } \\
\text { Occlusion Site }\end{array}$ & $\begin{array}{c}\text { Distal } \\
\text { Occlusion Site }\end{array}$ & $\begin{array}{l}\text { Thrombus } \\
\text { Length (mm) }\end{array}$ & $\begin{array}{l}\text { Clot Burden } \\
\text { Score }\end{array}$ & $\begin{array}{c}\text { Diameter of } \\
\text { Distal Vessel }(\mathrm{mm})\end{array}$ \\
\hline 1 & $R$ & Proximal M1 & Proximal M2 & $24.8 / 20.9^{a}$ & 6 & $1.0 / 1.7^{\mathrm{a}}$ \\
\hline 2 & L & Distal M1 & MCA bifurcation & 7.0 & 8 & 1.7 \\
\hline 3 & L & Distal M1 & Proximal M2 & 3.2 & 7 & 2.1 \\
\hline 4 & $\mathrm{R}$ & ICA terminus & Proximal $\mathrm{M} 1$ and $\mathrm{A} 1$ & 6.8 & 5 & 2.7 \\
\hline 5 & L & Cavernous ICA & MCA bifurcation & NA & 3 & 2.5 \\
\hline 6 & L & Cervical ICA & Supraclinoid ICA & NA & 7 & 3.7 \\
\hline 7 & L & Cervical ICA & ICA terminus & NA & 7 & 2.7 \\
\hline 8 & $\mathrm{R}$ & Distal M2 & Distal M2 & $9.6 / 6.1^{\mathrm{a}}$ & 9 & $1.2 / 1.8^{\mathrm{a}}$ \\
\hline 9 & $\mathrm{R}$ & Distal M2 & Distal M2 & 9.2 & 9 & 1.3 \\
\hline 10 & NA & Upper basilar trunk & L-P1 & 11.9 & NA & 2.6 \\
\hline
\end{tabular}

Note:-R indicates right; L, left; NA, not applicable.

a The clot is located in a bifurcation site, where there are 2 distal ends and subsequently 2 measurements for both thrombus length and diameter of the distal vessel.

Table 2: Quantitative and qualitative evaluation of collateral flow using time-resolved C-arm CBCTA data

\begin{tabular}{|c|c|c|c|c|c|c|c|c|}
\hline Case & $\begin{array}{l}\text { Acute } \\
\text { Occlusion } \\
\text { Site }\end{array}$ & $\begin{array}{c}\text { Flow } \\
\text { Direction } \\
\text { of } \mathrm{MCA}^{\mathrm{a}}\end{array}$ & $\begin{array}{l}\text { Measuring } \\
\text { Point }\end{array}$ & $\begin{array}{c}\text { Difference of } \\
\text { Time-to-Peak } \\
\text { (Lesion-Normal) }^{\mathrm{b}}\end{array}$ & $\begin{array}{c}\text { Relative } \\
\text { Peak Density } \\
\text { (Lesion/Normal) }^{c}\end{array}$ & $\begin{array}{l}\text { Score of } \\
\text { Tan et } \mathrm{al}^{13} \\
(0-3)\end{array}$ & $\begin{array}{c}\text { Score of } \\
\text { Miteff et al }{ }^{14} \\
\text { (3-Point) }\end{array}$ & $\begin{array}{c}\text { Score of } \\
\text { Maas et } \mathrm{al}^{4} \\
(1-5)\end{array}$ \\
\hline 1 & R-M1 & $R$ & Proximal M2 & $10 \sec (29-9)$ & $84.8 \%(302 / 356)$ & 3 & Good & 4 \\
\hline 2 & L-M1 & $R$ & Proximal M2 & $9 \sec (31-13)$ & $60.1 \%(292 / 486)$ & 2 & Good & 2 \\
\hline 3 & L-M1 & A & Proximal M2 & $1.5 \mathrm{sec}(10-7)$ & $90.8 \%(364 / 401)$ & 3 & Good & 3 \\
\hline 4 & R-ICA T & $\mathrm{R}$ & Proximal M1 & $8.5 \mathrm{sec}(34-17)$ & $58.6 \%(396 / 676)$ & 3 & Good & 3 \\
\hline 5 & L-ICA and $M 1$ & $R$ & Proximal M2 & $8 \sec (52-36)$ & $58.0 \%(341 / 588)$ & 1 & Good & 2 \\
\hline 6 & L-ICA & A & Distal M1 & $4 \mathrm{sec}(64-56)$ & $62.8 \%(301 / 479)$ & 3 & Good & 3 \\
\hline 7 & L-ICA & A & Distal M1 & $2.5 \mathrm{sec}(20-15)$ & $80.3 \%(309 / 385)$ & 3 & Good & 3 \\
\hline 8 & $\mathrm{R}-\mathrm{M} 2$ & NA & NA & NA & NA & NA & NA & NA \\
\hline 9 & $\mathrm{R}-\mathrm{M} 2$ & NA & NA & NA & NA & NA & NA & NA \\
\hline 10 & BA and L-P1 & NA & NA & NA & NA & NA & NA & NA \\
\hline
\end{tabular}

Note:-R indicates right; L, left; NA, not applicable; BA, basilar artery; T, terminus.

${ }^{a} A$ indicates antegrade; $R$, retrograde.

${ }^{b}$ Data in parentheses are the frame differences for time-to-peak. Each frame represents 0.5 seconds.

'Data in parentheses are the Hounsfield units of measured points, which represent the level of enhancement of the vessel.

stream vascular territory was detected in 3 patients. Analysis of time-density curves documented the late arrival of contrast by collateral flow with a difference of 1.5-10 seconds in time-to-peak and a reduction in peak density between $9.2 \%$ and $42 \%$. We believe that the latter reflects the reduced blood flow volume.

Two raters were in full agreement that the ability to evaluate clot burden and collateral flow with time-resolved C-arm CBCTA was equal to or better than that of the conventional techniques. They were also in good agreement that with the C-arm CBCTAs, additional information was available that could not be obtained from the conventional techniques (ie, clot distribution and dynamic information on collateral flow, $\alpha=1$ ). Therefore, the 2 raters preferred the CBCTAs for evaluation of clot burden and collateral flow $(\alpha=1)$.

\section{DISCUSSION}

In this feasibility study, we have demonstrated the ability to evaluate collateral status and clot burden by using time-resolved C-arm CBCTAs derived from C-arm CBCTP acquisitions. On the basis of our experience, this novel technique enabled us to evaluate collateral score and clot burden in a more comprehensive manner than currently available methods (ie, multidetector row CT or MRA). The availability of time-resolved C-arm CBCTAs and tMIP images that are derived from CBCTP acquisitions acquired at the site of treatment further enhances the angiographic suite as a one-stopshop for the care of patients with AIS.
Several modalities have been used for the evaluation of collateral status (eg, DSA, CT, MR imaging, and transcranial Doppler). Although DSA allows assessment of anatomic and dynamic features of collateral flow, it is usually reserved for those patients selected for endovascular treatment. Full evaluation of collaterals with DSA also requires the catheterization of multiple arteries for full collateral assessment. The advantage of the C-arm time-resolved conebeam CTA over 2D DSA is due to the following reasons: 1 ) the ability to provide a global (3D instead of 2D) view of collaterals with a single IV injection rather than by a series of intra-arterial injections, and 2) the ability to provide time-resolved conebeam CTAs viewable from any desired angle at the point of treatment. Previous studies have shown that conventional CTAs may underestimate the collateral flow, overestimate the clot burden, and lack dynamic information compared with time-resolved 4D-CTAs. ${ }^{15-17}$ 4D-CTA is only used occasionally due to its current limited availability. Compared with these $4 \mathrm{D}$ CTAs, C-arm CBCTAs derived from C-arm CBCTP acquisitions are fully time-resolved. C-arm time-resolved CBCTAs not only provide static (anatomic) information but also dynamic (blood flow) information about collateral flow. Temporal MIP images, a fusion of every single-phase image of time-resolved CTA, can also be generated from the time-resolved CBCTA data as shown in Figs 1 and $2 B$.

The availability of time-resolved CBCTAs at the point of treatment is, in our opinion, a clear advantage over conventional CTAs 

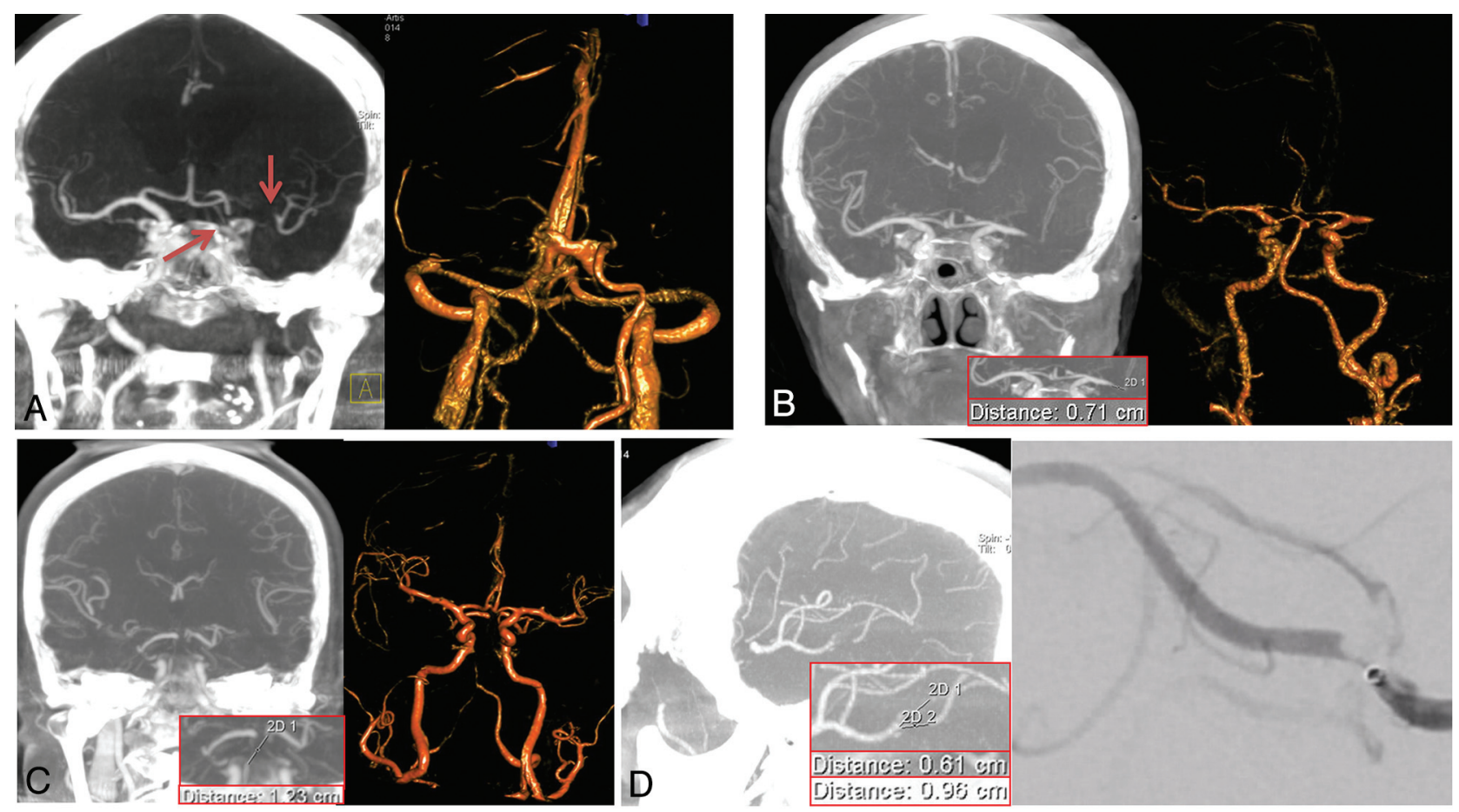

FIG 1. Coronal tMIP images ( $A$ left, $B$ left, and $C$ left) show a left ICA, a left M1, and an upper basilar trunk occlusion, respectively. The proximal and distal occlusion sites can be detected exactly from these images; this outcome enables the measurement of thrombus length in straight vessels. For the case with basilar tip occlusion, the thrombus distribution to the left Pl segment can be clearly seen ( $C$, left); this feature can facilitate the thrombectomy planning for this case. These occlusions can also be detected by the volume-rendered time-resolved $C$-arm CBCTA images at the late venous phase $(A$, right) or early arterial phase ( $B$, right and $C$, right). The sagittal tMIP image $(D$, left) shows an M2 occlusion at the bifurcation site, which was confirmed by a superselective injection through a microcatheter $(D$, right).

(the dynamic information about antegrade or retrograde collateral filling allowed us to see downstream filling in 3patients). If we had only conventional CTAs, this would not have been possible. These series were shown to be superior to conventional singlephase CTA for visualization of both collateral flow and clot burden. ${ }^{1,15}$ Using the time-resolved MIP images, we were able to determine the direction of blood flow (antegrade or retrograde) (Fig 2A) and also generate time-density curves, which provide some quantitative information about blood flow velocity and volume (Fig $2 C,-D$ ). Because they are acquired as an IV technique (thus including the full circulation), it seems likely that they will be preferable to intra-arterial DSA (with the requirement for multiple intra-arterial injections) as a means of acquiring this information. The C-arm CBCTAs have better spatial resolution than conventional CTAs and also provide whole-brain coverage, which is superior to most of the clinically used MDCTAs, ${ }^{18}$ which are either static if the whole-brain coverage is needed or only a timeresolved series for a thin slab of brain anatomy. The whole-brain coverage ensures isotropic reconstructions from at least the level of the ICA bifurcation.

Thus, it is possible to obtain secondary reformats that provide images from any direction. This flexibility of secondary reconstruction from any view angle should help to evaluate accurately the distribution of the clot burden and the size and angulation of distal vessels for any LVOs (Fig 1). This information can, in principle, facilitate accurate selection of the size and deploying site of a stent or thrombectomy device and could thus shorten the groin puncture-to-revascularization time. More important, time-re- solved C-arm CBCTA can be directly derived from the CBCTP data acquisition in the angiography suite, thus eliminating the need to transfer patients among different locations in the hospital. This change should result in a meaningful reduction of the picture-to-puncture time from stroke onset to endovascular treatment time. Because of its technical advantages and availability at the point of treatment, in our opinion, this novel imaging technique has great potential as a new tool for the care of patients with AIS suspected of having an LVO.

This study has several limitations. First, there was no control group. We were thus unable to directly compare the CBCTAs against other techniques. Because this was only a feasibility study, we are not able to offer rigorous comparisons of CBCTAs with either MDCTAs (single or multiphase) or MRAs. Second, our sample size was small; this feature further limits the ability to understand the real utility of CBCTAs. We are working to establish a prospective multicenter study, which will eliminate these limitations and will allow validation of the technique in realworld clinical practice.

\section{CONCLUSIONS}

In this small feasibility study, evaluation of clot burden and collateral status was feasible by using time-resolved C-arm CBCTAs derived from CBCTP acquisitions obtained in the angiography suite. Our results suggest that this novel technique provides a more complete method for evaluation of these parameters than conventional modalities. 

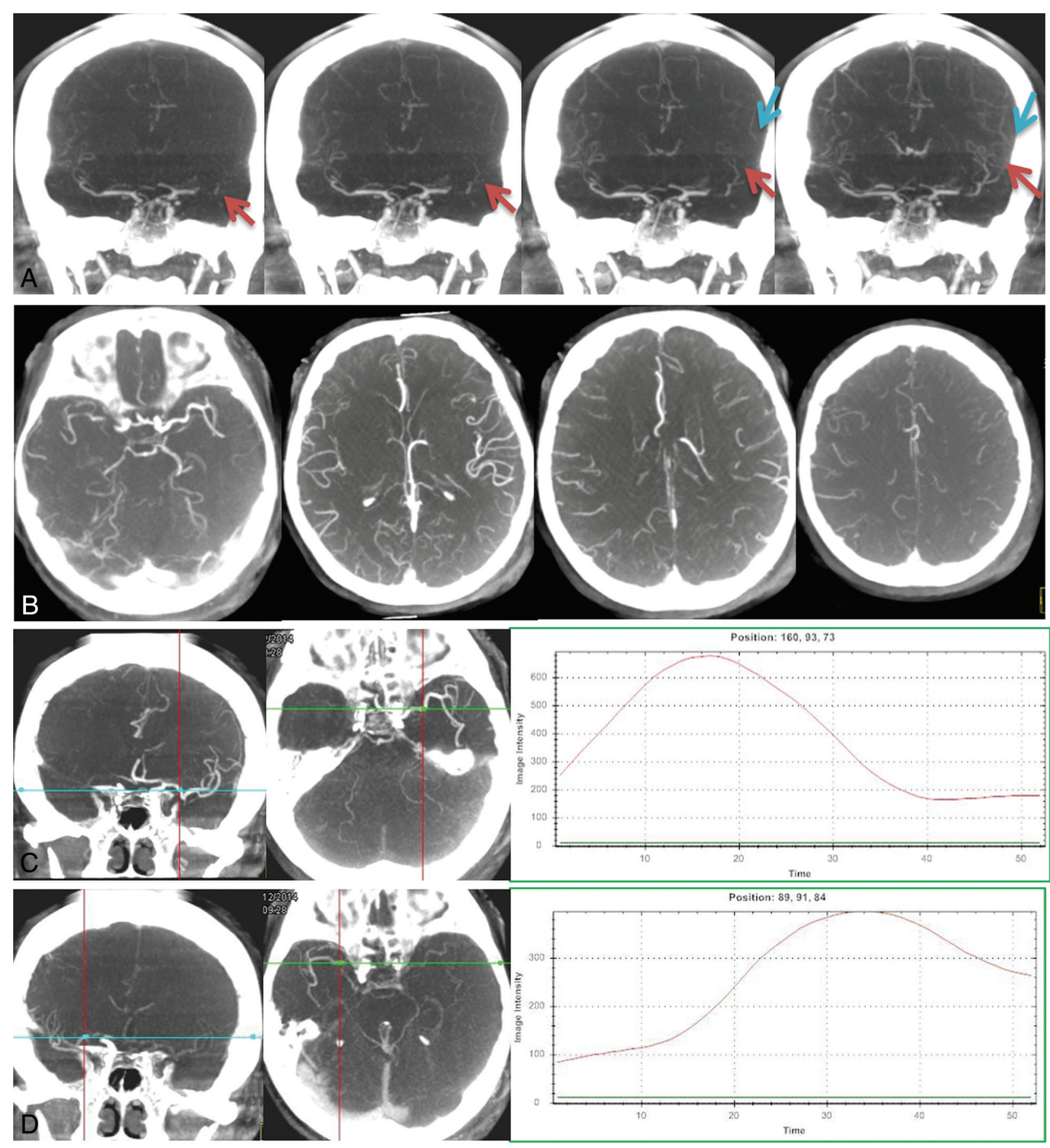

FIG 2. Antegrade flow for a case with left Ml occlusion was detected from time-resolved MIP images $(A)$, which may reflect partial recanalization of the vessel. The extent of collateral flow can be accurately detected from the tMIP images for a case with a right Ml occlusion (B). Collateral flow can be evaluated by using any of the 4 commonly used scoring systems based on these images. Time-density curves for bilateral symmetric measuring points can be generated from early-phase ( $C$, left and middle) and late-phase ( $D$, left and middle) time-resolved MIP images, respectively. Compared with the normal side (C, right), the lesion side (D, right) has delayed vessel filling (34/17, 8.5 seconds) and reduced vessel enhancement (396/676, 58.6\%).

\section{ACKNOWLEDGMENTS}

Research support provided by Siemens Healthineers USA.

Disclosures: Beverly Aagaard-Kienitz—UNRELATED: Grant: National Institutes of Health.* Charles Strother-RELATED: Support for Travel to Meetings for Study or Other Purposes: Siemens Healthineers; Provision of Writing Assistance, Medicines, Equipment, or Administrative Support: Siemens Healthineers, ${ }^{*}$ Comments: Equipment was provided under a Master Research Agreement between the University of Wisconsin School of Medicine and Public Health and Siemens Healthineers*; UNRELATED: Grant: National Institutes of Health*; Patents
(Planned, Pending, or Issued): Patents were filed by Wisconsin Alumni Research Foundation.* Guang-Hong Chen-UNRELATED: Grants/Grants Pending: National Institute of Biomedical Imaging and Bioengineering, Siemens Healthineers*; Royalties: patent royalty from GE Healthcare but not related to the work. * Money paid to the institution.

\section{REFERENCES}

1. Riedel CH, Zimmermann P, Jensen-Kondering U, et al. The importance of size: successful recanalization by intravenous thromboly- 
sis in acute anterior stroke depends on thrombus length. Stroke 2011;42:1775-77 CrossRef Medline

2. Singer OC, Berkefeld J, Nolte $\mathrm{CH}$, et al. Collateral vessels in proximal middle cerebral artery occlusion: the ENDOSTROKE study. Radiology 2015:274:851-58 CrossRef Medline

3. Bang OY, Saver JL, Kim SJ, et al. Collateral flow predicts response to endovascular therapy for acute ischemic stroke. Stroke 2011;42: 693-99 CrossRef Medline

4. Maas MB, Lev MH, Ay H, et al. Collateral vessels on CT angiography predict outcome in acute ischemic stroke. Stroke 2009;40:3001-05 CrossRef Medline

5. Campbell BC, Mitchell PJ, Kleinig TJ, et al; EXTEND-IA Investigators. Endovascular therapy for ischemic stroke with perfusion-imaging selection. N Engl J Med 2015;372:1009-18 CrossRef Medline

6. Goyal M, Demchuk AM, Menon BK, et al; ESCAPE Trial Investigators. Randomized assessment of rapid endovascular treatment of ischemic stroke. N Engl J Med 2015;372:1019-30 CrossRef Medline

7. Royalty K, Manhart M, Pulfer K, et al. C-arm CT measurement of cerebral blood volume and cerebral blood flow using a novel highspeed acquisition and a single intravenous contrast injection. AJNR Am J Neuroradiol 2013;34:2131-38 CrossRef Medline

8. Struffert T, Deuerling-Zheng Y, Kloska S, et al. Dynamic angiography and perfusion imaging using flat detector $\mathrm{CT}$ in the angiography suite: a pilot study in patients with acute middle cerebral artery occlusions. AJNR Am J Neuroradiol 2015;36:1964-70 CrossRef Medline

9. Yang P, Niu K, Wu Y, et al. Time-resolved C-arm computed tomographic angiography derived from computed tomographic perfusion acquisition: new capability for one-stop-shop acute ischemic stroke treatment in the angiosuite. Stroke 2015;46:3383-89 CrossRef Medline

10. Niu K, Yang P, Wu Y, et al. C-arm conebeam CT perfusion imaging in the angiographic suite: a comparison with multidetector CT perfusion imaging. AJNR Am J Neuroradiol 2016;37:1303-09 CrossRef Medline

11. Chen GH, Tang J, Leng S. Prior image constrained compressed sensing (PICCS): a method to accurately reconstruct dynamic CT images from highly undersampled projection data sets. Med Phys 2008;35:660-63 CrossRef Medline

12. Tang J, Xu M, Niu K, et al. A novel temporal recovery technique to enable cone beam CT perfusion imaging using an interventional C-arm system. Proc SPIE 2013;8668:86681A CrossRef

13. Tan IY, Demchuk AM, Hopyan J, et al. CT angiography clot burden score and collateral score: correlation with clinical and radiologic outcomes in acute middle cerebral artery infarct. AJNR Am J Neuroradiol 2009;30:525-31 CrossRef Medline

14. Miteff F, Levi CR, Bateman GA, et al. The independent predictive utility of computed tomography angiographic collateral status in acute ischaemic stroke. Brain 2009;132:2231-38 CrossRef Medline

15. Frölich AM, Schrader D, Klotz E, et al. 4D CT angiography more closely defines intracranial thrombus burden than single-phase CT angiography. AJNR Am J Neuroradiol. 2013;34:1908-13 CrossRef Medline

16. Frölich AM, Wolff SL, Psychogios MN, et al. Time-resolved assessment of collateral flow using 4D CT angiography in large-vessel occlusion stroke. Eur Radiol 2014;24:390-96 CrossRef Medline

17. Frölich AM, Psychogios MN, Klotz E, et al. Antegrade flow across incomplete vessel occlusions can be distinguished from retrograde collateral flow using 4-dimensional computed tomographic angiography. Stroke 2012;43:2974-79 CrossRef Medline

18. Kamran M, Nagaraja S, Byrne JV. C-arm flat detector computed tomography: the technique and its applications in interventional neuro-radiology. Neuroradiology 2010;52:319-27 CrossRef Medline 